\title{
Ultrafast dynamics of 2-thiouracil investigated by time-resolved Auger spectroscopy
}

\author{
F Lever $^{1+}$, D Mayer $^{1+}$, D Picconi2* ${ }^{2 *}$ J Metje $^{1}$, S Alisauskas ${ }^{3}$, F. Calegari ${ }^{3,4,5,6}$, \\ S Düsterer ${ }^{3}$, C Ehlert $^{7}$, R Feifel $^{8}$, M Niebuhr ${ }^{1}$, B Manschwetus $^{3}$, M Kuhlmann $^{3}$, \\ T Mazza ${ }^{9}$, M S Robinson ${ }^{1}$, R J Squibb ${ }^{8}$, A Trabattoni ${ }^{3,4}$, M Wallner $^{8}$, P Saalfrank ${ }^{2}$, \\ T J A Wolf ${ }^{10}$ and M Gühr ${ }^{1 *}$ \\ ${ }^{1}$ Institut für Physik und Astronomie, Universität Potsdam, 14476 Potsdam Germany \\ ${ }^{2}$ Institut für Chemie, Universität Potsdam, 14476 Potsdam Germany \\ ${ }^{3}$ Deutsches Elektronen Synchroton (DESY), 22607 Hamburg, Germany \\ ${ }^{4}$ Center for Free-Electron Laser Science (CFEL), DESY, 22607 Hamburg, Germany \\ ${ }^{5}$ The Hamburg Centre for Ultrafast Imaging, Universität Hamburg, 22761 Hamburg, Germany \\ ${ }^{6}$ Institut für Experimentalphysik, Universität Hamburg, 22761 Hamburg, Germany \\ ${ }^{7}$ Heidelberg Institute for Theoretical Studies, HITS gGmbH, 69118 Heidelberg, Germany \\ ${ }^{8}$ Department of Physics, Gothenburg University, SE-41296 Gothenburg, Sweden \\ ${ }^{9}$ European XFEL GmbH, 22869 Schenefeld, Germany \\ ${ }^{10}$ Stanford PULSE Institute, SLAC National Accelerator Laboratory, 94025 Menlo Park, USA \\ + Contributed equally \\ *E-mail: : david.picconi@uni-potsdam.de,mguehr@uni-potsdam.de
}

\begin{abstract}
We present time-resolved ultraviolet-pump x-ray probe Auger spectra of 2-thiouracil. An ultraviolet induced shift towards higher kinetic energies is observed in the sulfur $2 p$ Auger decay. The difference Auger spectra of pumped and unpumped molecules exhibit ultrafast dynamics in the shift amplitude, in which three phases can be recognized. In the first $100 \mathrm{fs}$, a shift towards higher kinetic energies is observed, followed by a $400 \mathrm{fs}$ shift back to lower kinetic energies and a 1 ps shift again to higher kinetic energies. We use a simple Coulomb-model, aided by quantum chemical calculations of potential energy states, to deduce a C-S bond expansion within the first $100 \mathrm{fs}$. The bond elongation triggers internal conversion from the photoexcited $S_{2}$ to the $S_{1}$ state. Based on timescales, the subsequent dynamics can be interpreted in terms of $S_{1}$ nuclear relaxation and $S_{1}$-triplet internal conversion.
\end{abstract}

\section{Introduction}

Nucleobases are strong absorbers in the ultraviolet (UV) domain and show ultrafast processes after photoexcitation. Canonical nucleobases exhibit ultrafast relaxation to the singlet and triplet ground states after UV excitation through internal conversion and intersystem crossing [1-4]. Electronic energy is efficiently transferred into vibrational excitation, a process that likely contributes to the remarkable stability of nucleic acids against UV-induced damage [1-3]. The processes underlying the ultrafast transitions violate the BornOppenheimer-Approximation (BOA), which allows for separate treatment of the electronic and nuclear degrees of freedom of a molecule, and is an important tool in modelling many molecular phenomena. In the case of close-lying potential energy surfaces such as avoided crossings or conical intersections (CIs), non-adiabatic coupling elements, which are neglected in the BOA, become large and lead to mixing of different Born-Oppenheimer electronic states. This redistributes the molecular population over initially unexcited potential energy surfaces [4,5]. The study of nucleobase dynamics, that cannot be described within the framework of the BOA, presents challenges both for theory as well as experiments that have been faced in the past by many systematic theoretical and experimental studies, reviewed for example in Refs. [1-3,7].

Thionucleobases are obtained by replacing one or two oxygen atoms by sulphur in canonical nucleobases, and exhibit two major differences compared to their canonical counterparts. The absorption spectrum is shifted from the UVC region into the UVA region [8,9], which is much more abundant on the earth surface. In addition, photoexcitation produces long living triplet states in thionucleobases, leading to cross linking $[10,11]$ and the creation of reactive singlet 
oxygen via a reaction with the triplet oxygen molecules in the vicinity $[12,13]$. These properties create on one hand a higher skin cancer risk for patients treated with thionucleobase medication for immunosuppression [14], on the other hand they might open the path for targeted photoinduced tumor therapy $[15,16]$.

The special case of thiouracil can be used for sketching the reaction pathway following UV excitation. The system is initially excited to the $S_{2}$ state, with ${ }^{1} \pi \pi^{*}$ electronic character. In solution, intersystem crossing has been determined to be on a few hundred femtosecond timescale in 2-thiouracil and 4thiouracil, as well as doubly thionated 2-4-thiouracil [9]. The $\mathrm{S}_{1}{ }^{1} n \pi^{*}$ state has been suggested to act as a doorway state to the lower lying triplet states in excited-state absorption studies in 2-thiouracil [13] as well as for 4-thiouracil. For the latter, the lifetime of the ${ }^{1} n \pi^{*}$ state has been indirectly determined to be $225 \mathrm{fs}$ in solution [13,17] by looking at the mismatch between the ${ }^{1} \pi \pi^{*}$ singlet decay and triplet rise times. In contrast, time-resolved photoelectron studies in the gas phase deduce an ${ }^{1} n \pi^{*}$ lifetime in 4-thiouracil of several picoseconds via fit of the photoelectron spectra [18]. This discrepancy is unlikely a solvent effect, as similar comparisons of gas and condensed phase techniques in 2-thiouracil give good agreements [19]. For 2-thiouracil, a joint theoreticalexperimental investigation using Dyson orbitals in conjunction with gas-phase photoelectron spectroscopy [20] deduced a very fast time constant of 50 fs for ${ }^{1} \pi \pi^{*}{ }_{-}{ }^{1} n \pi^{*}$ internal conversion and 500 fs for the ${ }^{1} n \pi^{*}$ lifetime; excited state absorption studies in condensed phase report on a similar ${ }^{1} n \pi *$ lifetime [9].

We now concentrate on the case of 2-thiouracil (2-TU), on which we perform our studies. The mechanism for sub-100 fs decay out of the initially photoexcited $S_{2}$ state as well as the ultrafast intersystem crossing from the $S_{1}{ }^{1} n \pi^{*}$ state into triplet states are illuminated by theoretical investigations (see Ref. [21] for a concise review). Cui and Fang suggest electronic states as well as crucial geometries for three different pathways, one of which includes the ${ }^{1} n \pi^{*}$ state, while the other paths involve transitions directly from the $S_{2}$ to triplet states. Calculations by Mai, Marquetand and González predict two different pathways for $S_{2}-S_{1}$ internal conversion, involving two different minima of the photoexcited state of 2-thiouracil [22]. One minimum is attributed to a ${ }^{1} \pi_{s} \pi_{6} *$ electronic character possessing a nearly planar geometry, while the other minimum is attributed to a ${ }^{1} \pi_{s} \pi_{2} *$ electronic character and it is reached via elongation and out-of-plane pyramidalization of the $\mathrm{C}-\mathrm{S}$ bond. In nonadiabatic surface-hopping calculations at the CASPT2 level by the same group, both minima are predicted to be transiently populated, although the majority of the molecular photoexcited population is predicted to pass through the ${ }^{1} \pi_{s} \pi_{6} *$ minimum within 60 fs to a conical intersection connecting it to the ${ }^{1} n \pi^{*}$ state, which then is populated for around 500 fs before decaying into triplet states
[23]. A second dynamics paper from the same group on this topic using $\mathrm{ADC}(2)$ comes to very similar results but a longer 250 fs ${ }^{1} \pi \pi^{*}{ }_{-}{ }^{n} n \pi^{*}$ transition times due to pyramidalization occurring in the ${ }^{1} \pi \pi^{*}$ state [24].

In this paper, we investigate the dynamics of 2-thiouracil using ultrashort x-ray probe pulses. Generally, time-resolved $\mathrm{x}$-ray spectroscopy has been proven to be a useful tool for the study of structural changes in isolated molecules [25]. The $\mathrm{x}-$ rays interact with core electrons of the molecule, whose binding energies are strongly dependent on the element. Thus, $x$-rays offer the advantage of an element-selective spectroscopic view on molecular dynamics. In addition, due to the narrow spatial confinement of core-electronic wavefunctions, the transitions are highly site-specific. In the gas phase, these advantages have been used to follow for instance nucleobase internal conversion [26], fragmentation [27], ring-opening [28] as well as dissociation [29].

We use ultrafast x-ray induced Auger decay to investigate 2-thiouracil. This method has been used before to investigate the internal conversion of thymine [30]. The key feature in this study was the change in molecular geometry on the photoexcited state. This can be deduced from the delaydependent kinetic energy shift in the Auger spectrum. We used the fact that the kinetic energy of emitted Auger electrons depends strongly on the local bond distance of the molecule around the core hole created by the x-ray probe pulse. Auger electron spectroscopy is particularly suited for the use with free-electron lasers (FELs), since nonresonant Auger processes are insensitive to the energy of the probing photon, rendering the method immune against the energy fluctuation inherent in the self-amplified spontaneous emission (SASE) process. Moreover, for a given x-ray energy, several Auger decays of different elements can be addressed separately by analyzing different kinetic energy ranges, allowing the simultaneous study of the dynamics for different locations in the molecule.

We use ultrashort $\mathrm{x}$-ray pulses to probe the sulfur $2 \mathrm{p}$ core electrons of UV-photoexcited 2-thiouracil. We measure a general shift of the $2 p$ Auger spectrum towards higher kinetic energies. By comparison to simulations, we deduce that the molecule does not return to the molecular ground state for the maximal delay of our measurements of 2 ps. On the transient Auger signal, we identify three different dynamic features. A first, sub-100 fs shift of the Auger band towards higher energies, followed by a 500 fs period with a slight shift towards lower energies and finally a picosecond modulation with a shift to higher kinetic energies. We show that the initial sub-100 fs modulation in the Auger spectrum can be clearly attributed to a C-S local bond elongation in the photoexcited $\mathrm{S}_{2}$ state as predicted in the theoretical literature [22-24]. The high-energy edge shifts to lower kinetic energies in this time interval and we show that an effective theoretical model can reproduce this trend. The later modulations in the Auger 
spectrum fit dynamics previously attributed to the $S_{1}$ relaxation and $S_{1}$ - triplet intersystem crossing.

\section{Methods}

The experiment was performed at the FLASH2 facility at DESY, where the newly built URSA-PQ (German: Ultraschnelle Röntgenspektroskopie zur Abfrage der Photoenergiekonversion in Quantensystemen, engl. Ultrafast $\mathrm{x}$-ray spectroscopy for probing photoenergy conversion in quantum systems) experimental chamber [31] built by the Potsdam group was connected to the FEL beamline FL24 (see Fig. 1).

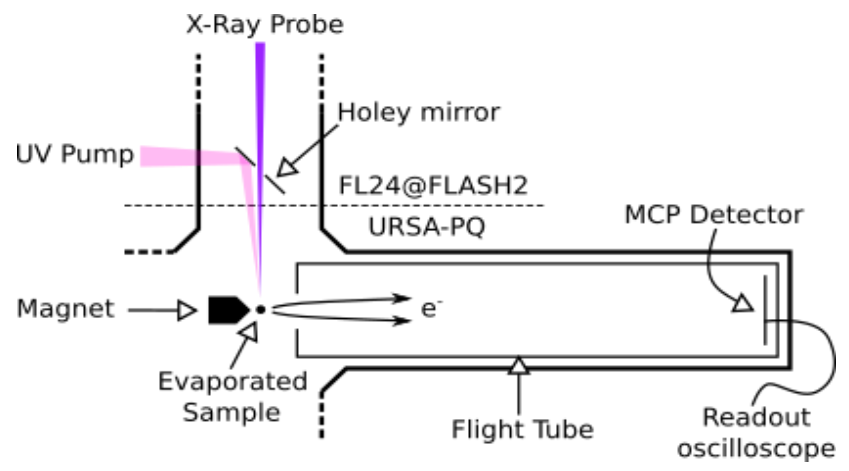

Figure 1. Experimental setup at the FL24 beamline of FLASH2. The UV pump and $\mathrm{x}$-ray probe beams from FLASH2 are focused into the URSA-PQ chamber and hit the sample in the interaction region of the magnetic bottle spectrometer. The sample is evaporated using a capillary oven (located above the interaction region, out of the plane of the diagram) [31]. The photo- and Auger electrons generated by the $\mathrm{x}$-ray interaction are guided into the flight tube by the magnetic bottle, detected using a multi-channel-plate (MCP) and their time of flight is measured by a fast analogue-digital converter (ADC).

The sample was introduced via a capillary oven [32] heated to a temperature of $150{ }^{\circ} \mathrm{C}$, creating a molecular gas jet that crosses the $\mathrm{X}$-ray beam in the interaction region of the TOF spectrometer. We have not observed degradation of the sample in previous experiments with our capillary oven under the conditions used. The $150{ }^{\circ} \mathrm{C}$ for sample evaporation have also been used in other studies on 2-thiouracil [18,33] as tautomerization can be neglected in this regime [33].

The molecule is preexcited with a $269 \mathrm{~nm}$ laser pulse at the maximum of its ${ }^{1} \pi \pi^{*}$ absorption band [18]. The pulse duration was about 80 fs as determined with a FROG (Frequency Resolved Optical Gating) setup. In the interaction region, the pulse was focused to a spot size of $50 \mu \mathrm{m}$ by means of a focusing mirror. Inside the beamline, the X-ray beam passed unperturbed through the centre of a holey mirror, whilst the UV beam was directed onto the reflective section of the holey mirror (just off to the side from the hole) to produce near- collinear trajectories for the two beams, as seen in Fig.1. The UV laser power was adjusted by means of a $\lambda / 2$ plate polarizer combination. In the experiments, the UV laser pulse energy was set below $1 \mu \mathrm{J}$. We performed systematic power scans on the time-dependent spectral features to make sure that the UV induced signal is not over-pumped.

The FEL produces frequency-tunable $\mathrm{x}$-ray pulses via SASE radiation. We used a mean photon energy of $272 \mathrm{eV}$ with a spectral bandwidth of $1-2 \%$ (including jitter), as determined by the photoemission of rare gases in electron time-of-flight spectrometers (eTOF) integrated into the beamline [34]. The x-ray beam was linearly polarized parallel to the axis of our magnetic bottle spectrometer and parallel to the UV polarization. The x-ray pulses were focused by means of Kirkpatrick-Baez (KB) mirrors to a spot size slightly larger than the UV spot. We used systematic power scans to make sure that the $\mathrm{x}$-ray induced electron spectra did not exhibit nonlinear phenomena.

The URSA-PQ setup contains a magnetic bottle electron spectrometer (MBES), providing high angular collection efficiency for electrons [35]. We used a permanent magnet and soft iron cone to reach a high magnetic field at the interaction region, which adiabatically transforms into a homogeneous magnetic field of a solenoid in a $1.7 \mathrm{~m}$ long flight tube. The magnet defines a small volume from which the electrons are guided into the flight tube. This volume is smaller than the optical beam overlap along the beam propagation axis. A retardation potential of $80 \mathrm{~V}$ was applied to the electrons using an electrostatic lens in front of the flight tube, diminishing their speed and increasing the time of flight and thus energy resolution.

The flight tube is kept at constant potential. At the very end, electrons are accelerated on the multi-channel-plate detector by a $300 \mathrm{~V}$ potential drop over $3 \mathrm{~mm}$. The amplified electron signal trace is fully digitized using a 12 bit analog-to-digital converter (ADC) with $2 \mathrm{Gs} / \mathrm{sec}$ sample rate. The amplified electron pulses have a FWHM of $10 \mathrm{~ns}$, typical flight times are on the order of $250 \mathrm{~ns}$ for the fast valence electrons and 410 ns for the slower Auger electrons. We chose to integrate the ADC-traces to obtain our spectra, as individual electron hits could not be distinguished in TOF regions of high signal strength. The spectra were subsequently converted to a kinetic energy scale.

Tests on the $\mathrm{Kr}$ MNN Auger lines at $0 \mathrm{~V}$ retardation demonstrated a MBES resolution $(\mathrm{E} / \triangle \mathrm{E})$ of 40 . This is not yet the maximum achievable resolution, as some $\mathrm{x}$-ray beamhalo problems at the beamline prevented us from positioning the magnet closer to the interaction region which would allow for a better optimised signal. We did not check the resolution at the retardation used further below $(80 \mathrm{~V})$. We estimate the resolution based on the sulphur $2 \mathrm{p}$ photoline with $3.5 \mathrm{eV}$ width at $103.5 \mathrm{eV}$ to be better than 30 with respect to the total kinetic energy or 7 relative to the retarded kinetic energy. The 
photoline itself is considerably broadened due to the FEL linewidth and therefore our estimate is really an upper limit.

Spatial overlap of the UV-pump and x-ray probe pulses was obtained by viewing a YAG screen inserted in the interaction region through a large work-distance microscope lens. Temporal overlap was established coarsely on a 50 ps scale using a fast photodiode and high-bandwidth (13 GHz) oscilloscope checking the rising flank of the UV and x-ray induced signals. Fine temporal overlap was found with the use of the sample itself, utilizing the time-dependent behavior of Auger and 2p-photoelectron lines. The width of the timeuncertainty function is $180 \mathrm{fs}$, time zero can be found with an accuracy of \pm 20 fs.

The FLASH2 FEL delivers pulse-trains with a frequency of $10 \mathrm{~Hz}$; we used 50 pulses at a $200 \mathrm{kHz}$ repetition rate in each pulse-train [36]. An eTOF spectrum is recorded and saved for each FEL pulse. The UV laser is set to run at $100 \mathrm{kHz}$ intraburst rep-rate, such that every second $\mathrm{x}$-ray shot is pumped by the optical laser, allowing the calculation of shotby-shot difference spectra. This results in $500 \mathrm{x}$-ray pulses per second, with 250 pulses UV pumped. In the rest of the discussion, we will refer to UV pumped shots as UV-on, while $\mathrm{x}$-ray only shots will be referred to as UV-off. FLASH2 instruments also provide a shot-by-shot measurement of the $\mathrm{x}$ ray pulse power by means of a combined ion-electron detector. This data has been used to rescale each eTOF spectra, therefore correcting for fluctuations in the SASE pulse power.

A bunch arrival monitor (BAM) at the FEL measures the time of arrival of each electron bunch, giving information on the jitter of the x-ray arrival time $[37,38]$. The width of the arrival time distribution has been measured to be $140 \mathrm{fs}$. The length of the x-ray pulse plus non-corrected jitter was estimated to be 160 fs. Pump-probe delays have been corrected with the BAM data and rebinned, exploiting the jitter and allowing finer delay binning than the time steps chosen during the measurement. The bin sizes are chosen by dividing the shot distribution in discrete parts, so that each bin is populated by the same number of shots. That is, the delay length of each bin is adjusted so that all bins have the same statistics. The delay points have been measured by random scans in repeated cycles over many hours. Thus any systematic drifts in the data-acquisition would average out.

To facilitate the interpretation of the experimental data, we make use of ab-initio quantum chemical calculations to explore the potential energy surfaces (PESs) of the neutral (2TU), core-ionized (2-TU+) and dicationic (2-TU++) molecule. The ground and excited states of 2-TU and 2-TU++ were computed using ground state coupled cluster theory with singles and doubles (CCSD) and the equation-of-motion formalism for the excitation energies (EOM-EE-CCSD) with the $6-311++\mathrm{G}^{* *}$ basis set [39]. In order to explore the high lying states of 2-TU++, potential energy cuts along the C-S bond distance were performed using time-dependent density functional theory (TDDFT) with the B3LYP functional and the $6-311++\mathrm{G}^{* *}$ basis set. Geometry optimizations for the ground and excited states of 2-TU were also performed at the (EOM-)CCSD/6-311++G** level without geometrical constraints using Q-Chem, and the stability was verified by frequency calculations with the smaller $6-31++\mathrm{G}^{* *}$ basis set. Stable minima were found for the states $S_{0}, S_{1}, S_{2}$ and $T_{1}$. The minimum of $S_{0}$ has a nearly planar structure. Furthermore, optimizations where the molecule was constrained to be planar were also carried out and additional (unstable) stationary points could be located for the states $S_{0}, S_{1}, S_{2}, T_{1}$ and $T_{2}$. The core-ionized states were computed using the equation-ofmotion ionization potential formalism (EOM-IP-CCSD) with the $6-311++\mathrm{G}^{* *}$ basis set for the $\mathrm{S}$ atom and the $6-31++\mathrm{G}$ basis for all other atoms; in order to simulate the ionization from the excited states of 2-TU, the reference (neutral) CCSD wavefunction was obtained starting from an unrestricted Hartree-Fock wavefunction optimized using the maximumoverlap-method (MOM) [40]. All calculations were performed using the package Q-Chem 4.4 [41]. Spin-orbit coupling, leading to a splitting of the core ionized states of about $1 \mathrm{eV}$ is not included in the calculations, instead we model photoionization from the px,py,pz orbitals.

\section{Results}

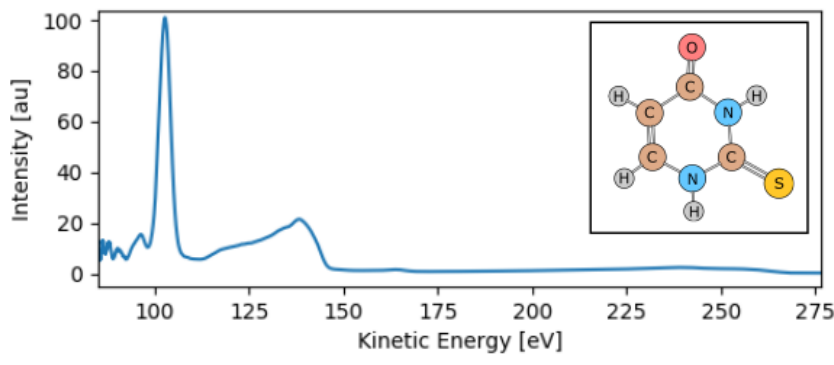

Figure 2. Overview of the photoemission spectrum of 2thiouracil at $272 \mathrm{eV}$ photon energy on an electron kinetic energy axis. Two main features are visible; the sulfur $2 p$ photoline centred at $103 \mathrm{eV}$ and the Auger band in the 115-150 eV range. Inset: structure of 2-thiouracil. 
The $272 \mathrm{eV}$ photons used in the experiment allow for an element and site-specific investigation of the molecular dynamics from the perspective of the sulfur atom. An overview of the electron kinetic energy spectrum without UV pre-excitation is shown in Fig. 2. Various features are visible in the graph. A sharp line at $103 \mathrm{eV}$ in kinetic energy, corresponding to a binding energy of $169 \mathrm{eV}$, is attributed to photoemission from the sulfur $2 p(j=3 / 2$ and $1 / 2)$ levels according to Ref. [33]. The $\sim 1 \mathrm{eV}$ spin-orbit splitting [33,42] cannot be resolved due to insufficient spectral resolution determined by the bandwidth of the SASE FEL and the MBES energy resolution. The neighboring feature, spanning the range $115-150 \mathrm{eV}$ is attributed to the Auger emission caused by the $2 p$ core vacancy. Scanning the photon energy does not change this feature, as it is typical for Auger decay. Furthermore, the energy levels with $2 p$ core hole binding energies around $170 \mathrm{eV}$ and ten to a few ten $\mathrm{eV}$ valence binding energies result in Auger features at this energy band. No detailed structure is visible, even at higher MBES resolution with higher retardations, due to many broad Auger decay channels overlapping. Coarsely, the atomic Auger spectrum should divide into three larger groups belonging to sulfur $3 \mathrm{p} 3 \mathrm{p} \rightarrow 2 \mathrm{p}$ (highest energy), $3 \mathrm{p} 3 \mathrm{~s} \rightarrow 2 \mathrm{p}$ and $3 \mathrm{~s} 3 \mathrm{~s} \rightarrow 2 \mathrm{p}$ (lowest energy) type Auger decays. For molecules with mixed atomic orbitals resulting in molecular orbitals, these groups can still be identified in case of oxygen Auger decay [30], here it is less pronounced.

In Fig. 3a), we show the pump-probe difference spectra in the energy range of the Auger features in false colorrepresentation. The spectra are obtained by subtracting UVoff shots from the UV-on shots, accumulating data from $~ 16$ million FEL shots over all delays. A positive differential signal (rendered with red color in the figures) signifies that UV pumping leads to more emitted electrons for that specific kinetic energy, while negative values (rendered with blue color in the figures) stand for a UV-induced depletion of the electron signal. Time zero was determined by maximizing the cross-correlation between a step function and the differential signal intensity. The delay values should therefore be regarded as relative to the appearance time of this feature.
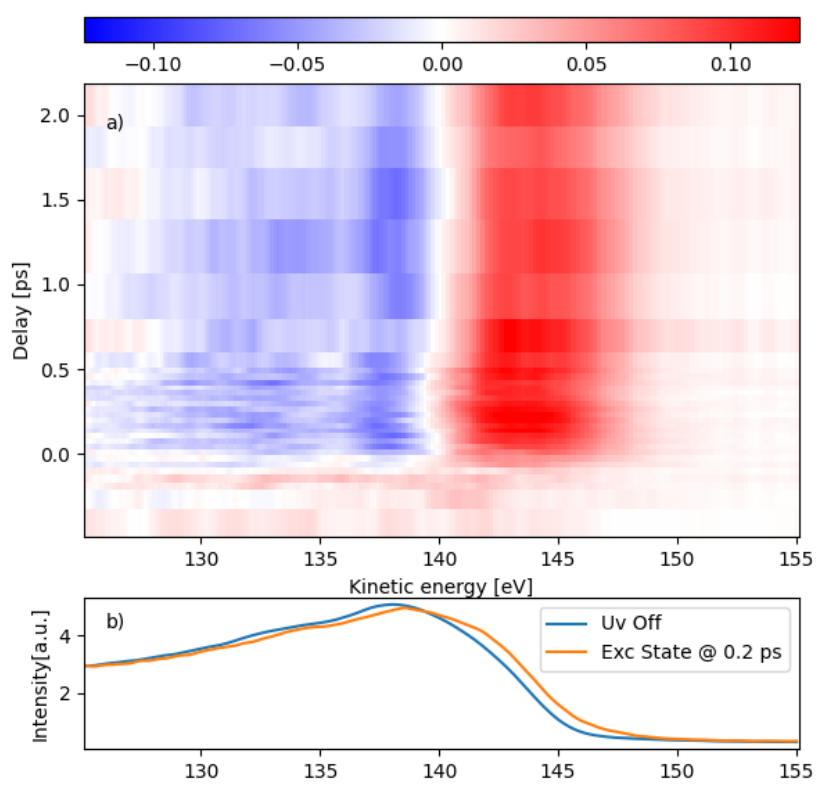

Figure 3. a) False-color representation of time resolved differential Auger spectrum (UV-on - UV-off) for 2-thiouracil (red: positive signal, blue: negative signal). A differential feature appears at $\mathrm{t} \sim-60 \mathrm{fs}$ and remains visible for all recorded delays. The Auger emission shifts to higher kinetic energies with UVillumination and ultrafast changes in the shape are visible around time zero. b) Spectra without UV excitation (Uv Off, blue) and spectrum of the excited state at 0.2 ps delay (orange) recovered by the procedure described in the text.

For positive delays, a general shift of the Auger spectrum toward higher kinetic energies is visible. This coarse shift of intensity in the difference spectrum related to the UV excitation is about $4 \mathrm{eV}$, however, the shift undergoes dynamics on an $\mathrm{eV}$ scale, as we will show below. We first investigate the coarse shift closer. The UV-on spectrum is given as $f^{*} E S+(1-f) G S$, where $E S$ and $G S$ are the excited state and ground state Auger spectra respectively and $f$ is the fraction of excited molecules. The UV-off spectrum is given as $G S$. The difference UV-on minus UV-off is thus $f(E S$-GS). In the process of photoexcitation, the $f$ changes.

An estimate of the excited state Auger spectrum is shown in Fig. 3b. It has been obtained from the UV-on and UV-off data. We need to model $f$ as a function of delay to obtain $E S$. Assuming that the fraction of excited molecules, $f$ introduced above, in the ensemble rises as an error function from 0 to 0.22 we succeeded to reconstruct the ES spectrum. The final $f$ of 0.22 was chosen as the minimum possible value that does not lead to negative amplitudes in the reconstructed excited spectrum ES. We identify that the shape of the Auger spectrum changes by comparing the UV-off spectrum with the reconstructed $E S$ spectrum at 200 fs delay. The maximum in the UV-off spectra is located at $138 \mathrm{eV}$. For the excited state spectrum at $200 \mathrm{fs}$, the region around the maximum flattens 
up to about $142 \mathrm{eV}$, for higher energies the excited state spectrum drops. This leads to the observed gross shift in the difference spectra with a bleach at $138 \mathrm{eV}$ and an increase from $140 \mathrm{eV}$ on with its maximum at $142 \mathrm{eV}$. The edge itself shifts only by about $1 \mathrm{eV}$ in the center of the edge and about 2 $\mathrm{eV}$ at the very high energy region. Assuming that the ES spectrum is completely static (no molecular dynamics after photoexcitation), the difference spectrum would not show any dynamics, apart from a changing scaling factor $f$. We however observe a fine shift visible in the zero line, thus indicating the presence of ultrafast processes in the relaxation pathway of the molecule.
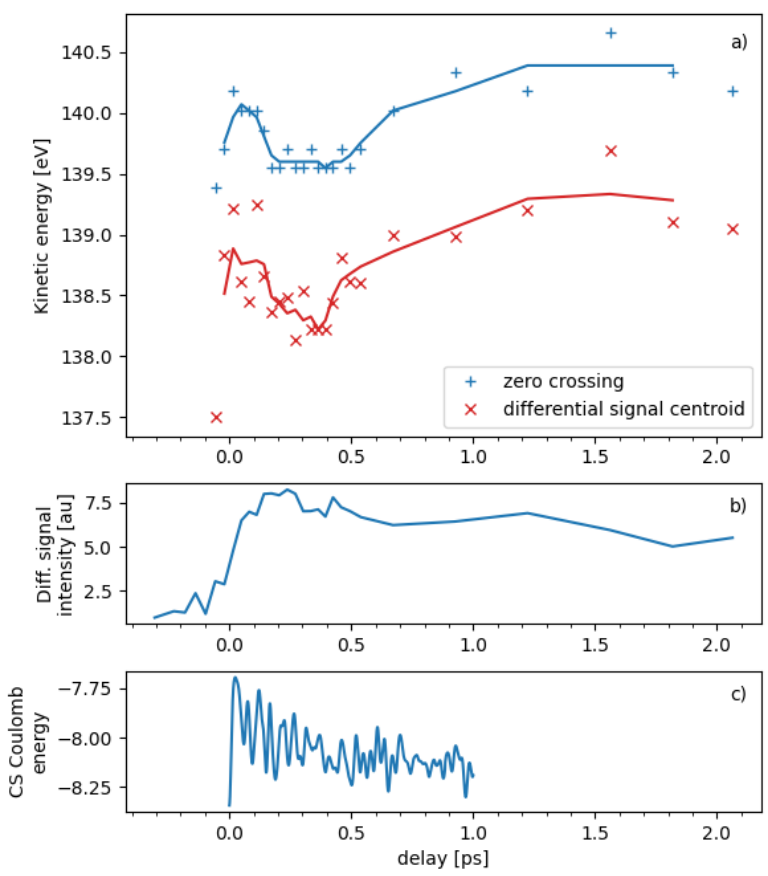

Figure 4. a) Zero crossing and differential signal centroid positions vs delay. Ultrafast dynamics is visible after time zero, with changes occurring on timescales from 100 fs to 1 ps. Solid lines are 3 point moving averages. b) Differential signal intensity, calculated from the cumulative sum of positive plus absolute of negative data points for each delay value. c)

Negative Coulomb energy constructed from the trajectory set of Ref. [24] using a simple Coulomb potential as a function of C-S distance.

In order to quantify the shift dynamics, the zero-crossing position of the differential signal (white line in between blue and red features) needs to be analyzed for each time delay. We estimated this parameter by maximizing the cross-correlation of the kinetic energy spectrum with a sign function. This method delivers the blue datapoints in Fig. 4a). In addition, we calculated the center of mass of the positive and negative contributions and averaged their value (red datapoints in Fig. 4a). The analysis of the centroid positions allows us to be sensitive to changes in the overall spectral distribution that might not affect the zero crossing point.

The aforementioned analysis technique does not give meaningful results when no differential features are present as it would be noise dominated. Therefore, we use the absolute intensity of the differential signal in Fig. 4b) (positive contribution + absolute of negative contribution) to select the delay regions that can be interpreted. The onset of the differential feature is found at delay $\sim-60 \mathrm{fs}$, and we clearly identify the typical lineshape in this time bin in the false color plot of Fig. 3a).

The Auger dynamics shows three phases (see Fig. 4a). First, a fast shift towards higher kinetic energies on a $\sim 100 \mathrm{fs}$ scale is visible, shifting the centroid difference as well as the zero crossing up by about $1 \mathrm{eV}$ in kinetic energy. Since the time resolution of the experiment is in this regime, we cannot give more precise estimates of the dynamics. After this initial rise in the kinetic energy, a transient dip of $\sim 0.5 \mathrm{eV}$ is visible, lasting for about 400 fs. This is followed by a 1 ps rise in centroid/zero crossing energy by $1 \mathrm{eV}$.

We now concentrate on the shift in the edge of the excited state Auger spectrum at high kinetic energies. We therefore analyze the time dependent ES spectra with the dynamic $f$ described above. Figure 5 shows the edge-position, defined by the ES signal being above $20 \%$ of the peak signal for each delay bin. The shifts are evaluated relative to the $E S$ spectrum at zero delay. Ultrafast dynamics on the same time scales as presented above can be discerned here, with some differences in the shift amplitudes.

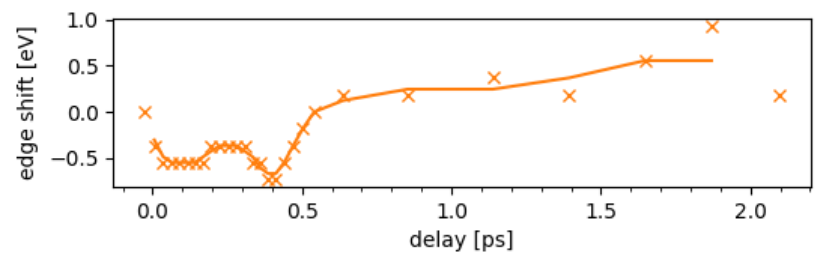

Figure 5. Auger edge shift as a function of pump-probe delay. Edge values are taken at the point where the signal rises over $20 \%$ of the peak intensity. Data points are shown for delays where the excited state population is above $1 \%$ of the final value. Solid line is a 3 point moving average.

The three phases mentioned above are discernible here again. The initial $100 \mathrm{fs}$ dynamics is characterized by a shift of the edge position of $-0.5 \mathrm{eV}$ (relative to the first datapoint), which is the opposite trend as shown in the zero position and centroid. This is followed by a plateau and a further dip at the 400 fs mark. After the dip, a fast $1 \mathrm{eV}$ blue shift is observed, persisting for the remaining of the time evolution. The different signs of spectral shift in the edge and at zero crossing 
in the beginning actually indicates that the excited state Auger spectrum undergoes changes in its shape.

\section{Discussion}

First, we discuss the results of the calculations. Table 1 reports our calculated energies at different stationary points on the excited states surfaces, and the corresponding value for the $\mathrm{C}$ $\mathrm{S}$ bond distance. Geometry optimizations have been carried out with and without the constraint of planarity. Local and global minima on the singlet and triplet potential energy surfaces of 2-TU have been located by Mai et al. using multistate complete active space perturbation theory calculations and in the present study using EOM-CCSD theory [22]. The calculated energies as well as geometry parameters are close to those of Mai et al. [21]. Surface-hopping trajectories calculated by Mai et al. predict that in the first 50 fs the molecule retains a near-planar geometry, and so do many trajectories at subsequent times and after transitions from $S_{2}$ to other states.

\begin{tabular}{|l|l|l|l|l|l|l|l|l|}
\hline & \multicolumn{3}{|l|}{ planar geometries } & \multicolumn{4}{|l|}{$\begin{array}{l}\text { non-planar } \\
\text { geometries }\end{array}$} \\
\hline minimum & $\mathrm{S}_{0} *$ & $\mathrm{~S}_{1} *$ & $\mathrm{~S}_{2} *$ & $\mathrm{~T}_{1} *$ & $\mathrm{~T}_{2} *$ & $\mathrm{~S}_{1}$ & $\mathrm{~S}_{2}$ & $\mathrm{~T}_{1}$ \\
\hline $\begin{array}{l}\text { energy } \\
{[\mathrm{eV}]}\end{array}$ & 0.00 & 3.78 & 4.44 & 3.17 & 3.65 & 3.48 & 3.96 & 3.00 \\
\hline $\begin{array}{l}\mathrm{C}-\mathrm{S} \\
\text { distance } \\
{[\AA]}\end{array}$ & 1.65 & 1.74 & 1.76 & 1.67 & 1.73 & 1.77 & 1.90 & 1.77 \\
\hline
\end{tabular}

Table 1. Calculated electronic energies and C-S bond distances at planar $(*)$ and non-planar minima of 2-thiouracil, optimized at the EOM-CCSD/6-311++G** level.

In order to investigate the initial behavior of the Auger signal at photoexcitation, we computed the energies of the valenceexcited, core-ionized and dicationic states in the FranckCondon region (see Fig. 6). We also calculated PESs along a reaction pathway which linearly interpolates between the unrestricted optimized minima of $S_{0}($ reaction coordinate $=0)$ and the initially photoexcited $S_{2}$ (reaction coordinate $=1$ ) states. This will help later in the interpretation of the high kinetic energy Auger edge.

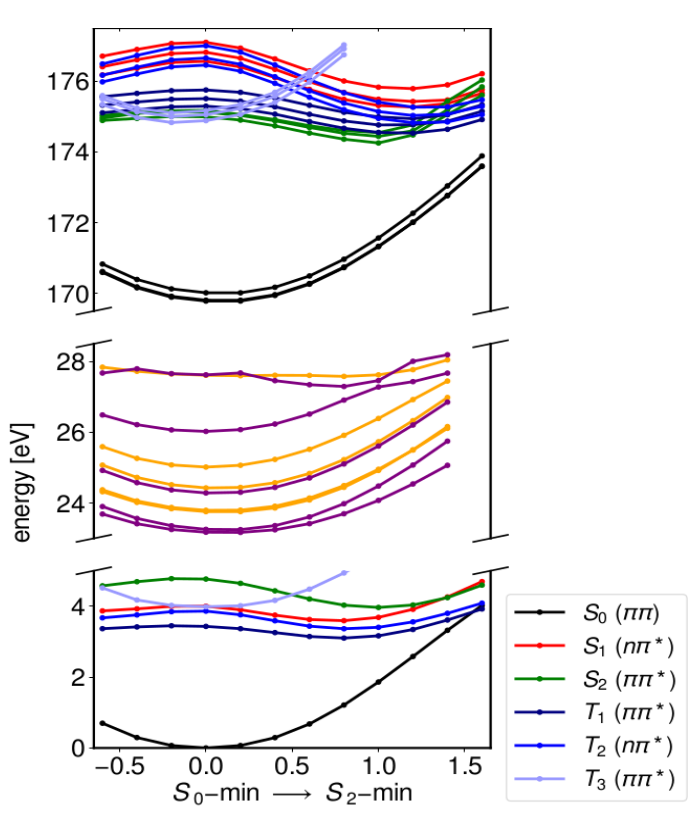

Figure 6. Calculated energy values for different electronic levels and for various nuclear configurations interpolated between the $\mathrm{S}_{0}$ and $\mathrm{S}_{2}$ minima. The energy scale is divided in ground and valence excited states (lower portion), 10 lowest energy dicationic states (middle) and core ionized $\left(2 \mathrm{p}^{-1}\right)$ states (upper). The core excited states follow the color coding of the valence states, meaning that their valence configuration is maintained with a $2 p$ core hole. The core ionized states of each valence state are divided into three states, according to px,py,pz ionization. SO coupling, leading to an observed core-ionized state splitting of $1 \mathrm{eV}$, is not included in our calculation. Singlet and triplet dicationic states are shown in orange and violet, respectively. Auger features with the highest observable kinetic energy can be calculated from the energy gap between core excited and dicationic states.

The data in the Franck-Condon region allows us to estimate the change in the kinetic energy of emitted Auger electrons, as Auger kinetic energy is equal to the difference between the core ionized state (one electron missing from the sulfur $2 p$ shell) and the dicationic manifold (with two electrons missing in the valence orbitals). Auger emission from the non-UV excited molecule occurs from the ground core-excited state (black around $170 \mathrm{eV}$ ) to many different dicationic states, of which only the lowest ones are calculated. Auger emission from the UV excited molecule occurs from the core ionized $\mathrm{S}_{2-}{ }^{1} \pi \pi^{*}$ state (green with an energy of $175 \mathrm{eV}$ in the FrankCondon region). Assuming that the rates for the Auger transitions do not change upon UV excitation, the model predicts a general shift towards higher kinetic energy of the Auger spectrum in the range of $5 \mathrm{eV}$.

Molecular dynamics on the core-ionized states can be neglected within the few fs-Auger decay timescale. All the 
states visited by the molecule after UV excitation show a gap with respect to the ground state. If efficient coupling to the ground state is absent on our measured timescales, as predicted by many previous papers $[9,20,21]$, the measured Auger spectra must show a long lasting blue shift that begins at time zero and remains present for all probed delays. This is in general agreement with the spectral trend observed in our measurements, and thus serves as a confirmation of excited state lifetimes longer than a few picoseconds.

The assumption of no Auger rate changes upon UV excitation however is oversimplified. As the valence occupation of two of the highest valence orbitals changes, there will be rate changing effects. Similar to the resonantAuger language, one can define spectator and participator channels here but the calculation of those rates is beyond the scope of this paper. We expect the valence reorganization effects induced by the UV excitation to be most effective at the high energy edge of the Auger spectrum, with participation from the highest orbitals. A comparison with the reconstructed ES spectrum (Fig. 3b) serves to support this point. The edge of the spectrum shows a UV induced shift of only 1-2 eV. In the region around the maximum, the redistribution of population is larger indicating shifts closer to those predicted by the gap between ground cationic state and excited cationic states, thus rate changes due to UV are less important in this region.

In order to interpret the initial ultrafast changes of the shift in the Auger spectrum, we include a model for the more energetic states of the dicationic manifold. The previously presented calculations on the lowest dicationic states will be used further below in a comparison to the edge features presented in Fig. 5. Upon sulfur 2p Auger decay, dicationic states with electrons missing in orbitals localized at the sulfur atom will be preferentially populated, as the decay matrix element is sensitive on the overlap of core and valence holes $[30,43]$. Those orbitals promote the C-S bonding and thus the Auger decay kinetic energy will be sensitive to the $\mathrm{C}-\mathrm{S}$ bond [30]. Since the missing valence electrons expose the nuclear charges, the Auger manifold will be dominated by Coulomb repulsion. The lowest energy dicationic states often show a binding character as seen in Fig. 6. We performed a Löwdin population analysis on the dicationic states of Fig. 6. Indeed, for most states the computed charge on the $\mathrm{S}$ atom is in the range $0.8-1.0 \mathrm{e}$. The electron hole on the $\mathrm{S}$ atom is present for all geometries, suggesting that Coulomb repulsion should be present, at least at longer $\mathrm{C}-\mathrm{S}$ distances. From the kinetic energy difference between the maximum and the edge of the Auger band, the Coulomb repulsive states should be located 7-8 eV above the lowest (bound) states of 2-TU2+, therefore they are not covered by our EOM-CCSD calculations. Repulsive potential energy curves in this energy range are predicted by TDDFT scans we performed along the C-S bond distance (not shown here). Indeed, with higher potential energy, dicationic states are increasingly dominated by Coulomb repulsion [44]. All statements below are therefore valid for the majority of the Auger decay channels, apart from the highest kinetic energy ones. Figure 7 shows a sketch of the electrostatic energy $e^{2} / R$ for a dicationic state as a function of $\mathrm{C}-\mathrm{S}$ bond stretch $R$. Qualitatively speaking, this can be seen as the central energy of the final state in the Auger decay process. Therefore, the decrease of Coulomb energy with increasing C$\mathrm{S}$ bond distance will lead to an increase in Auger kinetic energy, given that the core ionized starting state remains approximately constant in energy along the reaction coordinate as seen in Fig 6.

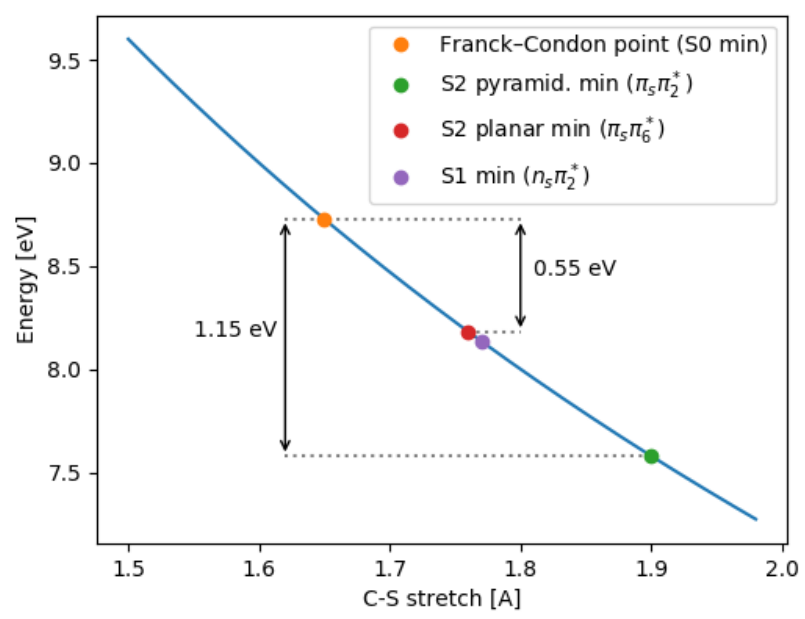

Figure 7. Electrostatic energy for a dicationic state for various bond lengths. Numerical values for the bond lengths as in Table 1. In the suggested dynamics, the electrostatic contribution to the molecular energy follows the highlighted path, caused by an ultrafast stretch and subsequent contraction of the $\mathrm{C}-\mathrm{S}$ bond.

The data on the zero-crossing as well as the centroid in Fig. 4 indicates an initial dynamic shift towards higher kinetic energies around time zero by less than $1 \mathrm{eV}$. Assuming that the ground and excited state spectra have similar shape, the overall spectral shift will be double the zero crossing shift, so between 1 and $2 \mathrm{eV}$. According to Fig. 7, the simple Coulombpotential model predicts a bond expansion. At the FranckCondon point, the molecule possesses a C-S bond distance of $1.65 \AA$ (Table 1). The experimentally observed shift would predict an expansion of the $\mathrm{C}-\mathrm{S}$ bond by nuclear relaxation into an interval from 1.85 to over $2 \AA$ within a delay range limited essentially by our temporal resolution. This interval does not include the calculated planar minimum at $1.76 \AA$ A. Our planar minimum has a structure similar to the (slightly nonplanar) $\pi_{s} \pi_{6} *$ minimum found by Mai et al. [23], and predicted to be the dominant pathway for nuclear relaxation out of the 
Franck-Condon region on the $\mathrm{S}_{2}$ surface at short times. The experimentally found interval includes the unrestricted minimum at $1.90 \AA$. This minimum has a structure similar to the $\pi_{s} \pi_{2} *$ minimum found by Mai et al. [22]. It is predicted to be populated, however with much less amplitude compared to the other minimum. The ADC(2) calculations however predict the internal conversion mostly occurring through a pyramidal deformation [24].

Shortly after the maximum in spectral shift, there is a reverse shift in the zero crossing and centroid towards lower kinetic energies by about $0.5 \mathrm{eV}$, as observed in Fig. 4. The feature is then stable for about 400 fs. The $\operatorname{ADC}(2)$ based trajectory calculations indeed show a reverse trend. In order to compare the results to our data, Mai et al. kindly sent us the $\mathrm{C}-\mathrm{S}$ distance of their trajectories [24]. We used those to calculate the dicationic energies and show them in Figure 4c). We clearly identify the reverse trend indicating shrinking C-S bond length over $400 \mathrm{fs}$. In the framework of the ADC(2) calculations, this period is dominated by dynamics on the $S_{2}$ state [24]. In comparison to these simulations, we seem to pick up the trends in the bond extension well. The rising kinetic energy after $400 \mathrm{fs}$ does not find its counterpart in any of the calculated C-S bond data of [24]. Thus it would need to be attributed to electronic state effects. On this timescale the triplet $\mathrm{T}_{1}$ state is taking over in [24].

The CASPT2 trajectory calculations of Mai et al. [23], display a $S_{2}-S_{1}$ internal conversion on the order of $60 \mathrm{fs}$ (below our time-resolution), with an $S_{1}$ state lifetime of 400-500 fs [23]. This interpretation is also chosen in previous experiments $[18,19,40]$. Afterwards, the $\mathrm{T}_{1}$ and $\mathrm{T}_{2}$ state become populated via an intersystem crossing with the $S_{1}$. In this framework, the fitting time scales suggests that the pumpprobe Auger signal in the interval between $50 \mathrm{fs}$ and $400 \mathrm{fs}$ is most likely due to the population in the $S_{1}\left({ }^{1} n \pi^{*}\right)$ state. According to the Coulomb-potential model, the decrease in kinetic energy should be associated with a slight contraction of the C-S bond. However, at the $\mathrm{S}_{1}$ minimum, optimized at the EOM-CCSD level, the C-S distance is $1.77 \AA$, i.e. very similar to the bond length at the $S_{2}$ planar minimum. Therefore, the C-S bond contraction could be explained by allowing that a relevant fraction of molecules on the $S_{2}$ surface either evolve towards the ${ }^{1} \pi_{s} \pi_{2}{ }^{*}$ minimum (a barrierless pathway as shown in Fig. 6, but less relevant according to the simulations of Ref. [23]) or undergo a large C-S elongation while retaining planarity, before undergoing the internal conversion. On the other hand, many of the nonadiabatic dynamics trajectories of Ref. [23] evolve along planar configurations also on the $S_{1}$ surface. Optimizing the $S_{1}$ state with the constraint of planarity we obtained a stationary point with a shorter C-S distance (1.74 $\AA$ ), which might also explain the observed decrease in kinetic energy. This model of course neglects all changes in the Auger spectra induced by electronic transitions. While our assumptions are justified on the initially photoexcited state, a transition from $S_{2}$ to $S_{1}$ can equally alter the Auger kinetic energies on the $\mathrm{eV}$ level. In order to make more precise statements about the competition of electronic vs. nuclear relaxation in shaping the Auger spectrum, further simulations need to be accomplished, which are beyond the scope of this work.

As stated above, we assumed that only nuclear geometry is able to influence the Auger kinetic energy. This is certainly justified for the initial relaxation of the molecular vibrational wave packet out of the Franck-Condon window. At later times, there might be an increasing influence of the electronic state changes on the Auger spectrum, and the argument will become more intertwined. However, we note that for Auger decay of the lighter oxygen, we have observed this strong electronic state dependence before as a massive redshift of the Auger band [30]. This is obviously lacking in our current experiment. We thus speculate, that the Auger decay of the much heavier sulfur, due to its increased density of states on the dicationic manifold will be less susceptible to those electronic state effects.

Regarding the dynamics of the high energy Auger edge, we can compare the excited state spectrum inferred from the experimental data to a synthetically constructed spectrum from the calculated potential energy curves of Fig. 6. It is important to note, that the computational results do not give us any information on the relative strengths of the various transitions; therefore, the constructed spectrum should be regarded as a qualitative guideline, and not as a predictive tool. Assuming a linear relaxation along the $S_{2}$ potential surface on a time scale of $60 \mathrm{fs}$, a time dependent excited state spectrum from the data in Fig. 6 can be constructed. The energy difference between the core excited $S_{2}$ state and each of the calculated dicationic states is taken as a function of time, and all the contributions are broadened and summed together to generate the time dependent spectrum. Convolving the result with an error function in order to model the non-simultaneous excitation of the ensemble, we obtain an estimate for the dynamics of the Auger edge. 


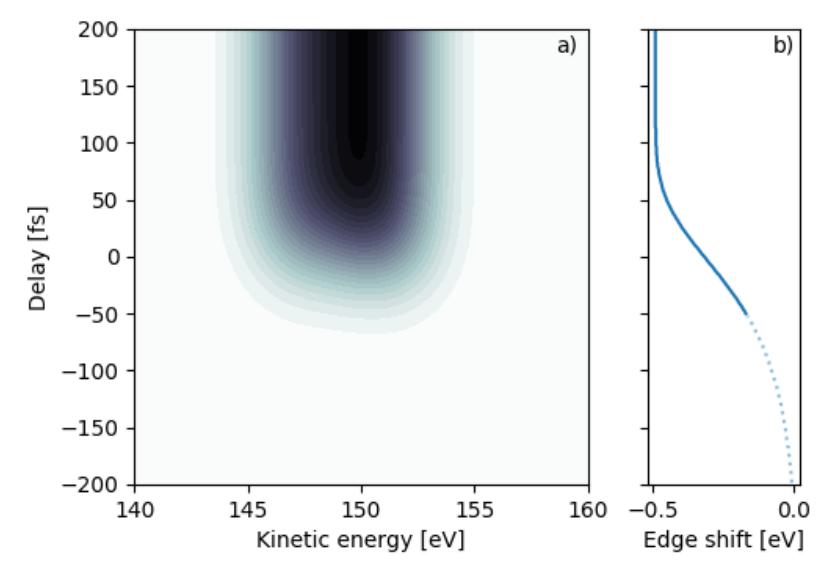

Figure 8. a) Reconstructed high kinetic energy band of the Auger spectrum for the calculated energy levels, convoluted with an error function to account for non-simultaneous excitation of the molecular ensemble. b) Auger edge shift in the reconstructed spectrum as a function of pump-probe delay. Dashed line shows values where the excited fraction is below 1\% (compare to Fig. 4b)

The calculated spectrum is shown in Fig. 8a. Analyzing the edge shift in the same way as in Fig. 5, we deduce a redshift of the edge position (Fig. 8b) in agreement with the experimental result in Fig. 5. The calculated value is $0.3 \mathrm{eV}$, while the experimental shift in the edge is $0.5 \mathrm{eV}$ with a discrete bin interval of $0.2 \mathrm{eV}$. According to the simulation, the energies of the lowest dicationic states and of the coreionized states accessed from $\mathrm{S}_{2}$ increase and decrease respectively, along the reaction pathway of Fig. 6. This is associated with a highest kinetic energy of the Auger electrons. Therefore, the short-time observed redshift of the Auger band edge provides further evidence for an ultrafast nuclear relaxation of the molecule out of the Franck-Condon region on a sub $100 \mathrm{fs}$ timescale.

On a longer time scale, for delays in the range 0.4 to $1.5 \mathrm{ps}$, the shift to higher kinetic energies observed on both the zero crossing and the edge position points to further ultrafast processes in the molecular dynamics (Fig. 4 and 5). The detailed interpretation of those features however requires a deeper understanding of the Auger processes in the molecule, due to the aforementioned electronic state effects.

\section{Summary}

In this work, we have used Auger spectroscopy to investigate the ultrafast dynamics of 2-thiouracil after UV excitation, using a UV pump - X-ray probe setup. The experiment has been carried out at beamline FL24 at the FLASH2 free electron laser. We observe a shift in the overall Auger emission pattern, as well as ultrafast dynamics in the shift amplitude, providing evidence for ultrafast processes in the molecule. The dynamics of the zero-crossing/centroid of Fig. 4 in conjunction with our quantum chemical calculations and the trajectory simulations by Mai et al. [23,24] strongly suggests that the time-resolved Auger signal is capable of capturing the main relaxation pathway of 2-thiouracil, which involves three steps: (i) an ultrafast, $<100 \mathrm{fs}$, process likely associated with an overstretching of the $\mathrm{C}-\mathrm{S}$ bond, which leads to the $S_{2}-S_{1}$ internal conversion; (ii) an intermediate evolution on the $S_{1}$ surfaces, for approximately 400 fs [23] or alternatively a shrinking $\mathrm{C}-\mathrm{S}$ bond dominated by $\mathrm{S}_{2}$ dynamics [24]; (iii) the final intersystem crossing and subsequent relaxation in the triplet manifold. The $\mathrm{C}-\mathrm{S}$ bond elongation in step (i) can be deduced from the observed zero line/centroid Auger shift towards higher kinetic energies using a simple Coulomb-model for dicationic states. Additional support for this behaviour is delivered by an observed edge shift towards lower kinetic energies, which is predicted by our model using the lowest dicationic states. Experimental evidence for (ii) and (iii) comes from an agreement of timescales in the zero line/centroid Auger shift, the details of the shift required additional Auger spectrum modelling involving electronic and nuclear dynamics, which is beyond the scope of this work.

\section{Acknowledgements}

We thank S. Mai (González group, Theoretical Chemistry, University of Vienna) for generously sending us trajectory data from Ref. [24] used in Fig. 4c). We thank the Volkswagen foundation for funding via a Lichtenberg Professorship. We thank the BMBF for funding the URSA-PQ apparatus and for funding J.M. via Verbundforschungsproject 05K16IP1. We acknowledge DFG funding via grants GU 1478/1-1 (MG) and SA 547/17-1 (PS). T.J.A.W was supported by the US Department of Energy, Office of Science, Basic Energy Sciences, Chemical Sciences, Geosciences, and Biosciences Division. We acknowledge DESY (Hamburg, Germany), a member of the Helmholtz Association HGF, for the provision of experimental facilities. Part of this research was carried out at FLASH2. F.C. acknowledges support from the European Research Council under the ERC-2014-StG STARLIGHT (Grant Agreement No. 637756). 


\section{References}

[1] M. Barbatti, A. C. Borin, and S. Ullrich, editors, Nucleobases in the Gas Phase and in Solvents (Springer, Cham, 2015).

[2] C. T. Middleton, K. de La Harpe, C. Su, Y. K. Law, C. E. Crespo-Hernández, and B. Kohler, Annu. Rev. Phys. Chem. 60, 217 (2009).

[3] C. Crespo-Hernandez, B. Cohen, P. Hare, and B. Kohler, Chem. Rev. 104, 1977 (2004).

[4] T. J. A. Wolf, R. M. Parrish, R. H. Myhre, T. J. Martínez, H. Koch, and M. Gühr, J. Phys. Chem. A 123, 6897 (2019).

[5] B. G. Levine and T. J. Martínez, Annu. Rev. Phys. Chem. 58, 613 (2007).

[6] D. R. Yarkony, Rev. Mod. Phys. 68, 985 (1996).

[7] R. Improta, F. Santoro, and L. Blancafort, Chem. Rev. 116, 3540 (2016).

[8] B. Ashwood, M. Pollum, and C. E. Crespo-Hernández, Photochem. Photobiol. 95, 33 (2019).

[9] M. Pollum, S. Jockusch, and C. E. Crespo-Hernández, Phys. Chem. Chem. Phys. 17, 27851 (2015).

[10] R. Brem, I. Daehn, and P. Karran, DNA Repair 10, 869 (2011). [11] X. Zhang, G. Jeffs, X. Ren, P. O’Donovan, B. Montaner, C. M. Perrett, P. Karran, and Y.-Z. Xu, DNA Repair 6, 344 (2007).

[12] H. Kuramochi, T. Kobayashi, T. Suzuki, and T. Ichimura, J. Phys. Chem. B 114, 8782 (2010).

[13] M. Pollum, L. Martínez-Fernández, and C. E. CrespoHernández, in Photoinduced Phenom. Nucleic Acids I, edited by M. Barbatti, A. C. Borin, and S. Ullrich (Springer International Publishing, Cham, 2014), pp. 245-327.

[14] S. Euvrard, J. Kanitakis, and A. Claudy, N. Engl. J. Med. 348, 1681 (2003).

[15] A. Massey, Y.-Z. Xu, and P. Karran, Curr. Biol. 11, 1142 (2001).

[16] O. Reelfs, P. Karran, and A. R. Young, Photochem Photobiol Sci 11, 148 (2012).

[17] R. Borrego-Varillas, D. C. Teles-Ferreira, A. Nenov, I. Conti, L. Ganzer, C. Manzoni, M. Garavelli, A. Maria de Paula, and G. Cerullo, J. Am. Chem. Soc. 140, 16087 (2018).

[18] A. Mohamadzade, S. Bai, M. Barbatti, and S. Ullrich, Chem. Phys. 515, 572 (2018).

[19] J. A. Sánchez-Rodríguez, A. Mohamadzade, S. Mai, B. Ashwood, M. Pollum, P. Marquetand, L. González, C. E. CrespoHernández, and S. Ullrich, Phys. Chem. Chem. Phys. 19, 19756 (2017).

[20] S. Mai, A. Mohamadzade, P. Marquetand, L. González, and S. Ullrich, Molecules 23, 2836 (2018).

[21] S. Arslancan, L. Martínez-Fernández, and I. Corral, Molecules 22, 998 (2017).

[22] S. Mai, P. Marquetand, and L. González, J. Phys. Chem. A 119, 9524 (2015).

[23] S. Mai, P. Marquetand, and L. González, J. Phys. Chem. Lett. 7, 1978 (2016).

[24] S. Mai, F. Plasser, M. Pabst, F. Neese, A. Köhn, and L. González, J. Chem. Phys. 147, 184109 (2017)

[25] L. Young, K. Ueda, M. Gühr, P. H. Bucksbaum, M. Simon, S. Mukamel, N. Rohringer, K. C. Prince, C. Masciovecchio, M.

Meyer, A. Rudenko, D. Rolles, C. Bostedt, M. Fuchs, D. A. Reis, R. Santra, H. Kapteyn, M. Murnane, H. Ibrahim, F. Légaré, M.
Vrakking, M. Isinger, D. Kroon, M. Gisselbrecht, A. L'Huillier, H. J. Wörner, and S. R. Leone, J. Phys. B At. Mol. Opt. Phys. 51, 032003 (2018).

[26] T. J. A. Wolf, R. H. Myhre, J. P. Cryan, S. Coriani, R. J. Squibb, A. Battistoni, N. Berrah, C. Bostedt, P. Bucksbaum, G. Coslovich, R. Feifel, K. J. Gaffney, J. Grilj, T. J. Martinez, S. Miyabe, S. P. Moeller, M. Mucke, A. Natan, R. Obaid, T. Osipov, O. Plekan, S. Wang, H. Koch, and M. Gühr, Nat. Commun. 8, 29 (2017).

[27] Y. Pertot, C. Schmidt, M. Matthews, A. Chauvet, M. Huppert, V. Svoboda, A. von Conta, A. Tehlar, D. Baykusheva, J.-P. Wolf, and H. J. Wörner, Science 355, 264 (2017).

[28] A. R. Attar, A. Bhattacherjee, C. D. Pemmaraju, K. Schnorr, K. D. Closser, D. Prendergast, and S. R. Leone, Science 356, 54 (2017).

[29] F. Brauße, G. Goldsztejn, K. Amini, R. Boll, S. Bari, C. Bomme, M. Brouard, M. Burt, B. C. de Miranda, S. Düsterer, B. Erk, M. Géléoc, R. Geneaux, A. S. Gentleman, R. Guillemin, I. Ismail, P. Johnsson, L. Journel, T. Kierspel, H. Köckert, J. Küpper, P. Lablanquie, J. Lahl, J. W. L. Lee, S. R. Mackenzie, S. Maclot, B. Manschwetus, A. S. Mereshchenko, T. Mullins, P. K. Olshin, J. Palaudoux, S. Patchkovskii, F. Penent, M. N. Piancastelli, D. Rompotis, T. Ruchon, A. Rudenko, E. Savelyev, N. Schirmel, S. Techert, O. Travnikova, S. Trippel, J. G. Underwood, C. Vallance, J. Wiese, M. Simon, D. M. P. Holland, T. Marchenko, A. Rouzée, and D. Rolles, Phys. Rev. A 97, 043429 (2018).

[30] B. K. McFarland, J. P. Farrell, S. Miyabe, F. Tarantelli, A. Aguilar, N. Berrah, C. Bostedt, J. D. Bozek, P. H. Bucksbaum, J. C. Castagna, R. N. Coffee, J. P. Cryan, L. Fang, R. Feifel, K. J.

Gaffney, J. M. Glownia, T. J. Martinez, M. Mucke, B. Murphy, A. Natan, T. Osipov, V. S. Petrović, S. Schorb, Th. Schultz, L. S. Spector, M. Swiggers, I. Tenney, S. Wang, J. L. White, W. White, and M. Gühr, Nat. Commun. 5, 4235 (2014).

[31] J.Metje et al., To be published (n.d.).

[32] B. K. McFarland, N. Berrah, C. Bostedt, J. Bozek, P. H.

Bucksbaum, J. C. Castagna, R. N. Coffee, J. P. Cryan, L. Fang, J. P. Farrell, R. Feifel, K. J. Gaffney, J. M. Glownia, T. J. Martinez, S. Miyabe, M. Mucke, B. Murphy, A. Natan, T. Osipov, V. S. Petrovic, S. Schorb, T. Schultz, L. S. Spector, M. Swiggers, F. Tarantelli, I. Tenney, S. Wang, J. L. White, W. White, and M. Gühr, J. Phys. Conf. Ser. 488, 012015 (2014).

[33] B. M. Giuliano, V. Feyer, K. C. Prince, M. Coreno, L. Evangelisti, S. Melandri, and W. Caminati, J. Phys. Chem. A 114, 12725 (2010).

[34] M. Braune, J. Buck, M. Kuhlmann, S. Grunewald, S. Düsterer, J. Viefhaus, and K. Tiedtke, J. Synchrotron Radiat. 25, 3 (2018).

[35] P. Kruit and F. H. Read, J. Phys. E 16, 313 (1983).

[36] B. Faatz, E. Plönjes, S. Ackermann, A. Agababyan, V. Asgekar, V. Ayvazyan, S. Baark, N. Baboi, V. Balandin, N. von Bargen, Y. Bican, O. Bilani, J. Bödewadt, M. Böhnert, R. Böspflug, S. Bonfigt, H. Bolz, F. Borges, O. Borkenhagen, M. Brachmanski, M. Braune, A. Brinkmann, O. Brovko, T. Bruns, P. Castro, J. Chen, M. K. Czwalinna, H. Damker, W. Decking, M. Degenhardt, A. Delfs, T. Delfs, H. Deng, M. Dressel, H.-T. Duhme, S. Düsterer, H. Eckoldt, A. Eislage, M. Felber, J. Feldhaus, P. Gessler, M. Gibau, N. Golubeva, T. Golz, J. Gonschior, A. Grebentsov, M. Grecki, C. Grün, S. Grunewald, K. Hacker, L. Hänisch, A. Hage, T. Hans, E. Hass, A. Hauberg, O. Hensler, M. Hesse, K. Heuck, A. Hidvegi, M. Holz, K. Honkavaara, H. Höppner, A. Ignatenko, J. Jäger, U. 
Jastrow, R. Kammering, S. Karstensen, A. Kaukher, H. Kay, B. Keil, K. Klose, V. Kocharyan, M. Köpke, M. Körfer, W. Kook, B. Krause, O. Krebs, S. Kreis, F. Krivan, J. Kuhlmann, M. Kuhlmann, G. Kube, T. Laarmann, C. Lechner, S. Lederer, A. Leuschner, D. Liebertz, J. Liebing, A. Liedtke, L. Lilje, T. Limberg, D. Lipka, B. Liu, B. Lorbeer, K. Ludwig, H. Mahn, G. Marinkovic, C. Martens, F. Marutzky, M. Maslocv, D. Meissner, N. Mildner, V. Miltchev, S. Molnar, D. Mross, F. Müller, R. Neumann, P. Neumann, D. Nölle, F. Obier, M. Pelzer, H.-B. Peters, K. Petersen, A. Petrosyan, G. Petrosyan, L. Petrosyan, V. Petrosyan, A. Petrov, S. Pfeiffer, A. Piotrowski, Z. Pisarov, T. Plath, P. Pototzki, M. J. Prandolini, J. Prenting, G. Priebe, B. Racky, T. Ramm, K. Rehlich, R. Riedel, M. Roggli, M. Röhling, J. Rönsch-Schulenburg, J. Rossbach, V. Rybnikov, J. Schäfer, J. Schaffran, H. Schlarb, G. Schlesselmann, M. Schlösser, P. Schmid, C. Schmidt, F. Schmidt-Föhre, M. Schmitz, E. Schneidmiller, A. Schöps, M. Scholz, S. Schreiber, K. Schütt, U. Schütz, H. Schulte-Schrepping, M. Schulz, A. Shabunov, P. Smirnov, E. Sombrowski, A. Sorokin, B. Sparr, J. Spengler, M. Staack, M. Stadler, C. Stechmann, B. Steffen, N. Stojanovic, V. Sychev, E. Syresin, T. Tanikawa, F. Tavella, N. Tesch, K. Tiedtke, M. Tischer, R. Treusch, S. Tripathi, P. Vagin, P. Vetrov, S. Vilcins, M. Vogt, A. de Z. Wagner, T. Wamsat, H. Weddig, G. Weichert, H. Weigelt, N. Wentowski, C. Wiebers, T. Wilksen, A. Willner, K. Wittenburg, T. Wohlenberg, J. Wortmann, W. Wurth, M. Yurkov, I. Zagorodnov, and J. Zemella, New J. Phys. 18, 062002 (2016). [37] S. Schulz, I. Grguraš, C. Behrens, H. Bromberger, J. T. Costello, M. K. Czwalinna, M. Felber, M. C. Hoffmann, M. Ilchen, H. Y. Liu, T. Mazza, M. Meyer, S. Pfeiffer, P. Prędki, S. Schefer, C. Schmidt, U. Wegner, H. Schlarb, and A. L. Cavalieri, Nat. Commun. 6, 5938 (2015).

[38] E. Savelyev, R. Boll, C. Bomme, N. Schirmel, H. Redlin, B. Erk, S. Düsterer, E. Müller, H. Höppner, S. Toleikis, J. Müller, M. Kristin Czwalinna, R. Treusch, T. Kierspel, T. Mullins, S. Trippel, J. Wiese, J. Küpper, F. Brauße, F. Krecinic, A. Rouzée, P. Rudawski, P. Johnsson, K. Amini, A. Lauer, M. Burt, M. Brouard, L. Christensen, J. Thøgersen, H. Stapelfeldt, N. Berrah, M. Müller, A. Ulmer, S. Techert, A. Rudenko, and D. Rolles, New J. Phys. 19, 043009 (2017).

[39] A. I. Krylov, Annu. Rev. Phys. Chem. 59, 433 (2008).

[40] N. A. Besley, A. T. B. Gilbert, and P. M. W. Gill, J. Chem. Phys. 130, 124308 (2009).

[41] Y. Shao, Z. Gan, E. Epifanovsky, A. T. B. Gilbert, M. Wormit, J. Kussmann, A. W. Lange, A. Behn, J. Deng, X. Feng, D. Ghosh, M. Goldey, P. R. Horn, L. D. Jacobson, I. Kaliman, R. Z.

Khaliullin, T. Kuś, A. Landau, J. Liu, E. I. Proynov, Y. M. Rhee, R. M. Richard, M. A. Rohrdanz, R. P. Steele, E. J. Sundstrom, H. L. Woodcock, P. M. Zimmerman, D. Zuev, B. Albrecht, E. Alguire, B. Austin, G. J. O. Beran, Y. A. Bernard, E. Berquist, K. Brandhorst, K. B. Bravaya, S. T. Brown, D. Casanova, C.-M. Chang, Y. Chen, S. H. Chien, K. D. Closser, D. L. Crittenden, M. Diedenhofen, R. A. DiStasio, H. Do, A. D. Dutoi, R. G. Edgar, S. Fatehi, L. FustiMolnar, A. Ghysels, A. Golubeva-Zadorozhnaya, J. Gomes, M. W. D. Hanson-Heine, P. H. P. Harbach, A. W. Hauser, E. G.

Hohenstein, Z. C. Holden, T.-C. Jagau, H. Ji, B. Kaduk, K.

Khistyaev, J. Kim, J. Kim, R. A. King, P. Klunzinger, D. Kosenkov, T. Kowalczyk, C. M. Krauter, K. U. Lao, A. D. Laurent, K. V. Lawler, S. V. Levchenko, C. Y. Lin, F. Liu, E. Livshits, R. C. Lochan, A. Luenser, P. Manohar, S. F. Manzer, S.-P. Mao, N. Mardirossian, A. V. Marenich, S. A. Maurer, N. J. Mayhall, E.
Neuscamman, C. M. Oana, R. Olivares-Amaya, D. P. O’Neill, J. A. Parkhill, T. M. Perrine, R. Peverati, A. Prociuk, D. R. Rehn, E. Rosta, N. J. Russ, S. M. Sharada, S. Sharma, D. W. Small, A. Sodt, T. Stein, D. Stück, Y.-C. Su, A. J. W. Thom, T. Tsuchimochi, V. Vanovschi, L. Vogt, O. Vydrov, T. Wang, M. A. Watson, J. Wenzel, A. White, C. F. Williams, J. Yang, S. Yeganeh, S. R. Yost, Z.-Q. You, I. Y. Zhang, X. Zhang, Y. Zhao, B. R. Brooks, G. K. L. Chan, D. M. Chipman, C. J. Cramer, W. A. Goddard, M. S. Gordon, W. J. Hehre, A. Klamt, H. F. Schaefer, M. W. Schmidt, C. D. Sherrill, D. G. Truhlar, A. Warshel, X. Xu, A. Aspuru-Guzik, R. Baer, A. T. Bell, N. A. Besley, J.-D. Chai, A. Dreuw, B. D. Dunietz, T. R. Furlani, S. R. Gwaltney, C.-P. Hsu, Y. Jung, J. Kong, D. S. Lambrecht, W. Liang, C. Ochsenfeld, V. A. Rassolov, L. V. Slipchenko, J. E. Subotnik, T. Van Voorhis, J. M. Herbert, A. I. Krylov, P. M. W. Gill, and M. Head-Gordon, Mol. Phys. 113, 184 (2015).

[42] Al Thompson and Doug Vaughan, X-RAY Data Booklet (Center for X-ray Optics and Advanced Light Source Lawrence, Berkeley National Laboratory, 2009).

[43] H. Siegbahn, L. Asplund, and P. Kelve, Chem. Phys. Lett. 35, 330 (1975)

[44] J. P. Cryan, J. M. Glownia, J. Andreasson, A. Belkacem, N. Berrah, C. I. Blaga, C. Bostedt, J. Bozek, N. A. Cherepkov, L. F. DiMauro, L. Fang, O. Gessner, M. Gühr, J. Hajdu, M. P. Hertlein, M. Hoener, O. Kornilov, J. P. Marangos, A. M. March, B. K. McFarland, H. Merdji, M. Messerschmidt, V. S. Petrović, C. Raman, D. Ray, D. A. Reis, S. K. Semenov, M. Trigo, J. L. White, W. White, L. Young, P. H. Bucksbaum, and R. N. Coffee, J. Phys. B At. Mol. Opt. Phys. 45, 055601 (2012). 